\title{
CHARGE Association With Choanal Atresia and Inner Ear Hypoplasia in a Child With a De Novo Chromosome Translocation t $(2 ; 7)(\mathbf{p} 14 ; \mathbf{q} 21.11)$
}

\author{
Donna M. Martin, ${ }^{1}$ Susan Sheldon, ${ }^{2}$ and Jerome L Gorski ${ }^{1,3 *}$ \\ ${ }^{1}$ Department of Pediatrics and Communicable Diseases, University of Michigan Medical School, Ann Arbor, Michigan \\ ${ }^{2}$ Department of Pathology, University of Michigan Medical School, Ann Arbor, Michigan \\ ${ }^{3}$ Department of Human Genetics, University of Michigan Medical School, Ann Arbor, Michigan
}

\begin{abstract}
A 3-year-old boy was diagnosed with CHARGE association on the basis of bilateral choanal atresia, absence of the semicircular canals, hypoplastic cochleae, genital hypoplasia, growth and developmental delays, cranial nerve dysfunction, and facial anomalies. Ophthalmologic and cardiac evaluations were normal. He was found to have an apparently balanced $t(2 ; 7)(\mathbf{p 1 4} ; \mathbf{q} 21.11)$ chromosomal translocation. Parental karyotypes were normal. Although there is evidence suggesting a genetic basis for CHARGE association, individuals with chromosomal abnormalities and CHARGE are rare. In the described patient, the presence of characteristic CHARGE features suggests that the $t(2 ; 7)(p 14 ; q 21.11)$ translocation breakpoints may cause a deletion or disruption of genes within the involved regions that are involved in the generation of the CHARGE association phenotype. (c) 2001 Wiley-Liss, Inc.
\end{abstract}

KEY WORDS: CHARGE; chromosome; choanal atresia; semicircular canals; cochleae; deafness

\section{INTRODUCTION}

CHARGE $(\mathrm{C}=$ coloboma,$\quad \mathrm{H}=$ heart disease, $\mathrm{A}=$ atresia of the choanae, $\mathrm{R}=$ retarded growth and development and/or CNS anomalies, $\mathrm{G}=$ genital hypoplasia, $\mathrm{E}=$ ear anomalies and/or deafness) is a nonran-

Grant sponsor: State of Michigan Department of Health; Grant number: 38-6006309.

*Correspondence to: Jerome L Gorski, Division of Pediatric Genetics, Rm 3570 Medical Science Research Bldg II, Box 0688, University of Michigan Medical School, Ann Arbor, MI 481090688. E-mail: jlgorski@umich.edu

Received 31 August 2000; Accepted 24 October 2000

Published online 22 January 2001 dom association of multiple congenital anomalies first described by Hall [1979]. The CHARGE acronym, proposed by Pagon et al. [1981], established the diagnostic criteria of CHARGE association as colobomata and/or choanal atresia and a total of at least 4 of the 6 major features. Subsequent evaluation of CHARGE individuals demonstrated characteristic facial features, renal abnormalities and cranial nerve dysfunction [Davenport et al., 1986, Oley et al., 1988]. More recently, a strikingly high frequency of semicircular canal atresia has been found among individuals with CHARGE [Tellier et al., 1998]. Most cases of CHARGE association are sporadic with an unknown etiology. However, familial cases and monozygotic twins with CHARGE suggest an underlying genetic mechanism. Few cases of CHARGE association with chromosomal rearrangements have been reported. Here we report a three-year-old boy with bilateral choanal atresia, absence of the semicircular canals, hypoplastic cochleae, genital hypoplasia, growth and developmental delays, facial anomalies, and cranial nerve dysfunction. He also had a de novo apparently balanced $t(2 ; 7)(p 14 ; q 21.11)$ chromosomal translocation, raising the possibility of a causative relationship between the chromosomal breakpoints and his congenital anomalies.

\section{CLINICAL REPORT}

C.Z. weighed $3370 \mathrm{~g}$ at birth, and was born after a 37week gestation to non-consanguineous parents, a 29year-old gravida 3 woman and a 30 -year-old father. The couple's two previous pregnancies resulted in a liveborn delivery and a first trimester miscarriage, respectively. The family history was negative for congenital birth defects. The pregnancy was complicated by an abnormal ultrasound at 18 weeks gestation that suggested nuchal thickening and enlarged lateral cerebral ventricles. Cytogenetic analysis of amniotic fluid showed an apparently balanced translocation between the short arm of chromosome 2 and the long arm of chromosome 7 with a chromosomal constitution of $46, \mathrm{XY}, \mathrm{t}(2 ; 7)$ (p14;q21.11). Parental karyotypes were normal. Serial ultrasounds showed a gradual resolution of the nuchal 
thickening and ventricular dilation. Labor and delivery were unremarkable and the newborn infant had a occipitofrontal circumference (OFC) of $36.6 \mathrm{~cm}$ and a length of $53 \mathrm{~cm}$.

Multiple medical problems were noted in the immediate newborn period. Bilateral choanal atresia required stent placement and surgical repair (Fig. 1). A head CT scan showed normally sized ventricles without additional structural brain anomalies. Brainstem auditory-evoked potentials were abnormal. Renal ultrasound showed mild left hydronephrosis with Grade II ureteral reflux. Transient hypoventilation responded to theophylline. Additional medical problems were experienced in the first year. Gastroesophageal reflux and recurrent aspiration pneumonia required fundoplication and gastrostomy tube placement. Bronchoscopy showed aberrant branching of the right pulmonary bronchus. Transient, low cortisol levels resolved with a subsequently normal endocrinologic evaluation (normal ACTH, testosterone, growth hormone, insulin-like growth factor I, thyroid function tests, and gonadotropin function). An umbilical hernia repair and an orchidopexy were performed.

Examination at 7 weeks showed normal growth parameters at the 25th percentile with an OFC of $37.7 \mathrm{~cm}$, a length of $55 \mathrm{~cm}$, and a weight of $4.04 \mathrm{~kg}$. The

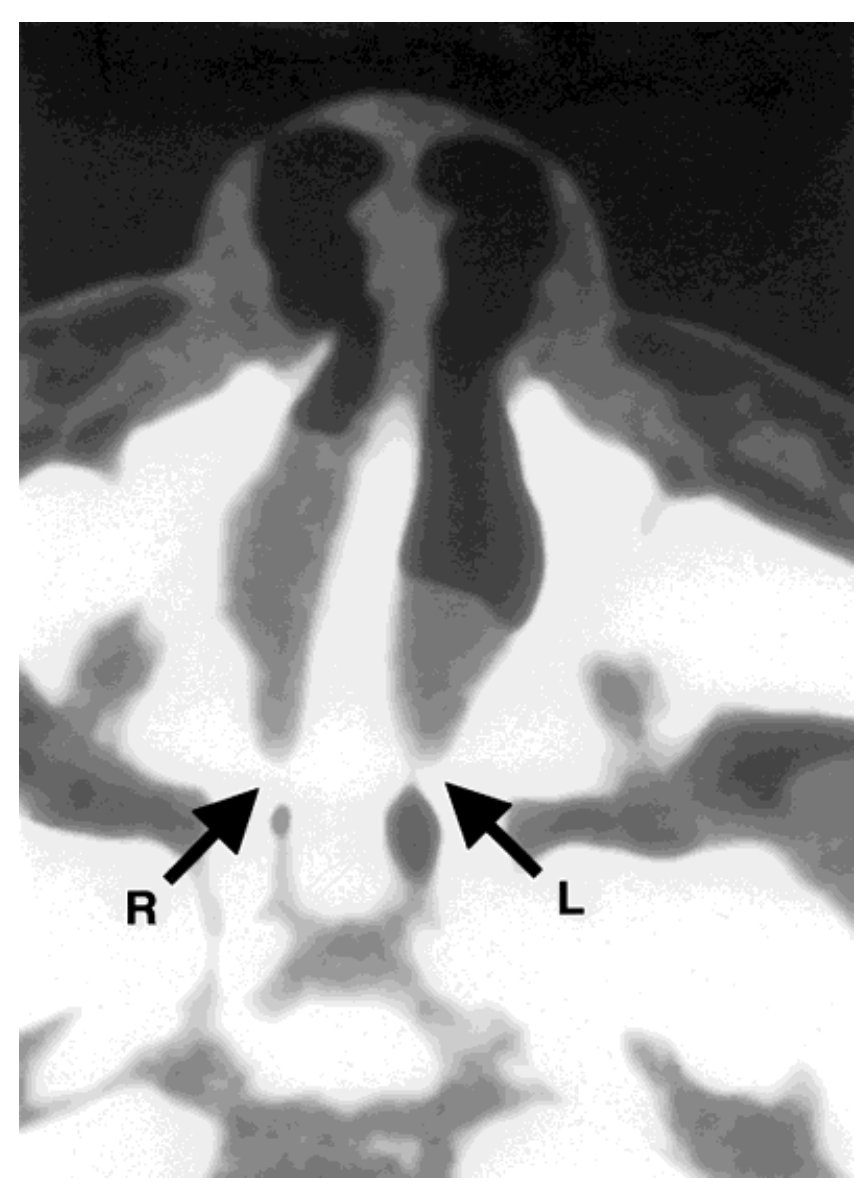

Fig. 1. Head CT (axial view) performed at 2 days shows bilateral bony choanal atresia (arrows).

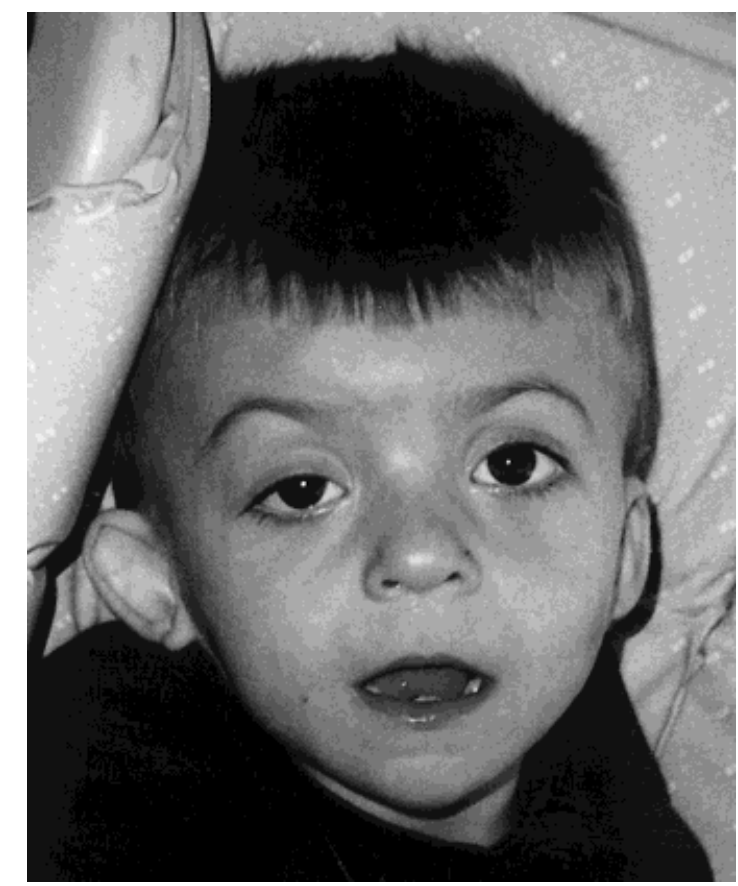

Fig. 2. A photograph of the patient at 3 years shows facial features, including bilateral ptosis, anteriorly protruding right ear, small, upturned nose, long philtrum, thin upper lip and mild micrognathia.

examination was remarkable for several minor dysmorphic facial features, including bilateral ptosis with intermittent right strabismus, a broad nasal bridge, posteriorly rotated ears with over-folded and cupped helices, and mild micrognathia. The neck was webbed with extra nuchal skin. The chest was asymmetric with the right nipple more rostrally placed than the left. Mild diastasis recti was present. The phallus was small and the scrotum was hypoplastic; the right testicle was undescended. The upper extremities were normal; metatarsus adductus was present. Examination at 13 months showed normal growth parameters at the 1025th percentile. Neurologic exam showed decreased muscular tone. Receptive and expressive speech and the acquisition of major gross motor signs were delayed: independent walking was attained at 42 months. Growth delays were apparent at 26 months when his weight $(10.6 \mathrm{~kg})$ was below the 5 th percentile for a 26 month-old and below the 50th percentile for a 13month-old, with a height $(82.7 \mathrm{~cm})$ also below the 5 th percentile for a 26-month-old and below the 50th percentile for a 19-month-old. Bone age performed at age 26 months showed dysharmonious bone age between 9 and 24 months. At age 3, he was noted to have minor facial anomalies, including ptosis, small, upturned nose, long philtrum, and thin upper lip (Fig. 2). A temporal bone CT scan showed an absence of the semicircular canals and bilaterally hypoplastic cochleae (Fig. 3).

Repeated dilated ophthalmologic exams failed to identify intraocular abnormalities. Repeat echocardiograms showed the heart to be structurally normal. With the exception of the anomalies noted above, radio- 

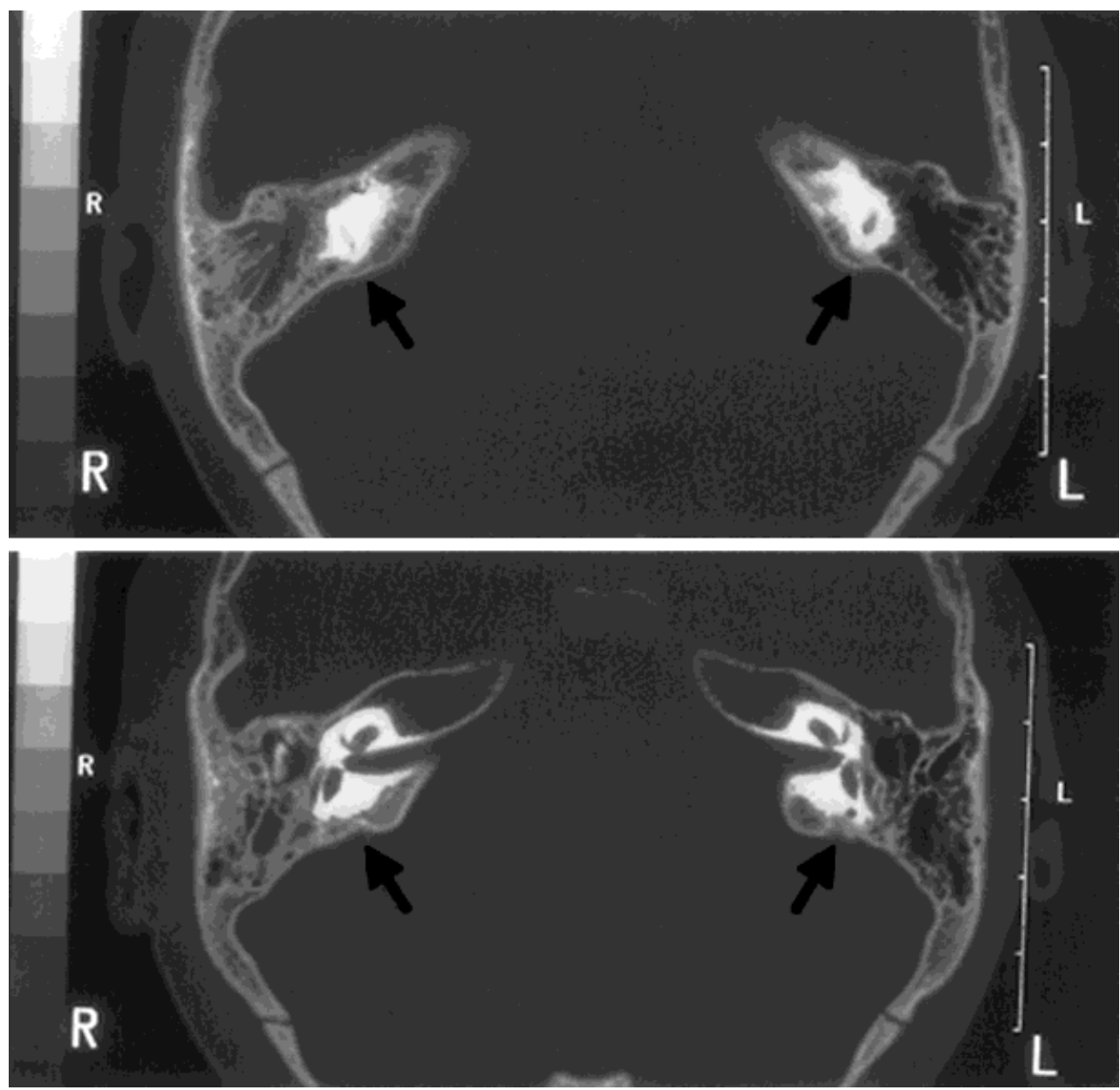

Fig. 3. Head CT of the temporal bone performed at age 32 months, demonstrating hypoplastic cochleae (upper panel) and semicircular canals (lower panel). Arrows in upper panel point to the existing two turns of the left and right cochleae, consistent with a Mondini anomaly. Arrows in lower panel point to small, irregular vestibules, with absence of the posterior and lateral semicircular canals. There are rudimentary, dysmorphic superior semicircular canals (lower panel).

graphic studies demonstrated a structurally normal skeleton. A peripheral blood karyotype confirmed the apparently balanced translocation involving chromosomes 2 and 7 (Fig. 4): karyotype 46,XY,t(2;7)(p14; q21.11).

\section{DISCUSSION}

Our patient's clinical features were diagnostic of CHARGE association. He had bilateral choanal atresia, growth and developmental delays, genital hypoplasia, and atresia of the semicircular canals and cochleae. His clinical features were within the original core criteria for a diagnosis of CHARGE [Pagon et al., 1981], which include coloboma or choanal atresia and four of the six cardinal features. Notably, atresia of the semicircular canals was recently demonstrated to be a prominent finding in CHARGE individuals [Tellier et al., 1998]. The patient also had a history of central hypoventilation requiring theophylline, grade II hydronephrosis, and a history of cranial nerve dysfunction manifested as swallowing and respiratory difficulties. Further evaluation of CHARGE individuals has revealed char- acteristic facial features and a high frequency of renal and cranial nerve abnormalities such as those seen in our patient [Oley et al., 1988; Tellier et al., 1998]. Our patient also met the revised diagnostic criteria for CHARGE, as proposed by Blake et al. [Blake et al., 1998]. Specifically, he had three major criteria (choanal atresia, characteristic ear abnormalities, and cranial nerve dysfunction) and three minor criteria (genital hypoplasia, developmental delay, and growth deficiency) for CHARGE. Facial features common to our patient and other individuals with CHARGE are striking (see Fig. 1 of Blake et al., 1998), and include facial asymmetry, ptosis, thin upper lip, and external ear anomalies. Our patient was also found to have a de novo chromosomal translocation $\mathrm{t}(2 ; 7)(\mathrm{p} 14 ; \mathrm{q} 21.11)$ that appeared balanced on cytogenetic analysis. To our knowledge, this is the first report of a child with CHARGE association and an apparently balanced chromosomal translocation.

In the majority of cases, CHARGE association occurs as a sporadic event of unknown cause. Rare familial cases, including concordance among monozygotic twins 

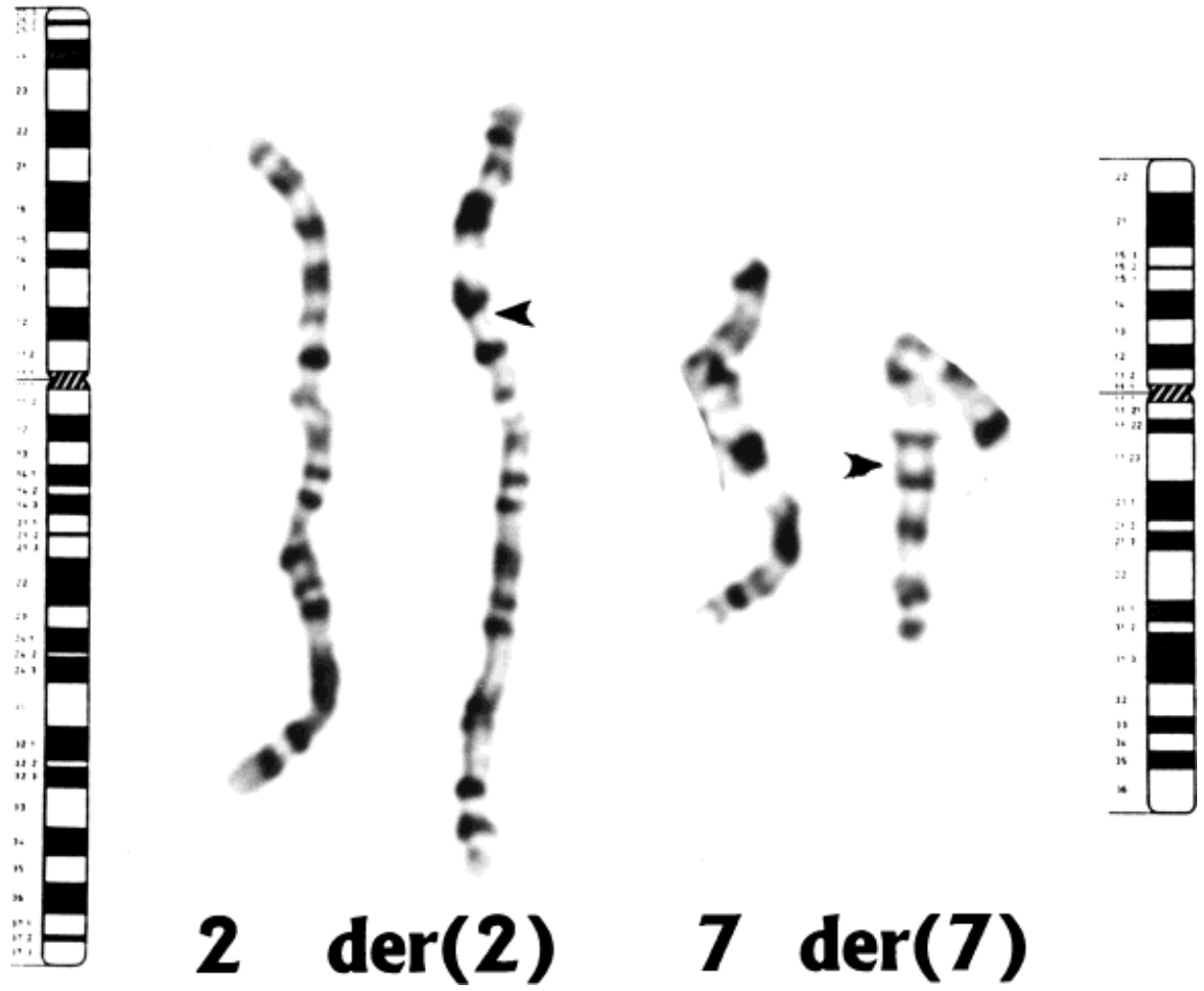

Fig. 4. Partial karyotype showing the normal chromosome 2, the derivative chromosome 2 with the breakpoint at p14 (arrow), the normal chromosome 7 , and the derivative chromosome 7 with the breakpoint at q21.11 (arrow).

and discordance among dizygotic twins, suggest that CHARGE association is a genetic condition [Mitchell et al., 1985; Metlay et al., 1987; Hall and Blumberg, 1988; Blake et al., 1989; Tellier et al., 1998]. Increased paternal age at conception has been documented in one series of CHARGE individuals, raising the possibility of a causative dominant mutation or submicroscopic deletion [Tellier et al., 1996]. Despite these findings, chromosomal rearrangements have been reported in only a handful of patients with CHARGE association. Clinical features of CHARGE association, including coloboma, choanal atresia, and congenital heart disease, have been reported in several children with DiGeorge sequence with chromosome $22 \mathrm{q} 11$ deletions [Clementi et al., 1991; Devriendt et al., 1998]. These cases highlight the overlap in phenotype between some individuals with CHARGE association and chromosome 22q11 deletions, and likely reflect genetic heterogeneity within the velocardiofacial/DiGeorge syndrome region on chromosome 22q11. Other chromosomal regions have been implicated in CHARGE association, including duplication of region $14 q 22 q 24.3$ in a child with iris coloboma, ventricular septal defect, soft tissue choanal atresia, growth retardation and sensorineural deafness [North et al., 1995]. These features, although consistent with CHARGE, are atypical in that colobomata in CHARGE more commonly involve the choroid, retina, or optic disk, and choanal atresia is more commonly bony and membranous [Tellier et al., 1998]. Hurst et al. [1991] identified a girl with bilateral retinal colobomata, tetralogy of Fallot, unilateral choanal atresia, sensorineural hearing loss, and a de novo balanced whole-arm translocation between the short and long arms of chromosomes 6 and $8: \mathrm{t}(6 ; 8)(\mathrm{p} 10 ; \mathrm{p} 10)$. However, in their patient, the proximity of the breakpoints to the centromeres of both chromosomes 6 and 8 decreases the likelihood of a causative effect, since centromeres are generally gene poor.

Chromosomal translocations, which appear balanced on standard cytogenetic analyses, may in some cases be associated with cryptic deletions near the breakpoints [Kumar et al., 1998]. In addition to the t(2;7) translocation visible on standard karyotype analysis, our patient may have had a separate, underlying genetic mechanism that contributed to the formation of his anomalies. Thus, some other locus distant to the identified $t(2 ; 7)$ translocation may contain a sequence alteration, not detectable by standard cytogenetic analyses, which contributed to our patient's features. Review of the literature revealed other reports of congenital anomalies associated with chromosome $7 q$. While none of these cases matches our patient exactly, shared features include congenital deafness [Ignatius et al., 1996], facial anomalies [Lopez-Rangel et al., 1993], and genital anomalies [Tajara et al., 1989]. We were unable to find any reports in the literature on individuals with small rearrangements of chromosome band $2 \mathrm{p} 14$.

In summary, we report a boy with a specific subset of CHARGE association features and a balanced trans- 
location between chromosomes 2 and 7. These findings are consistent with a relatively small chromosomal abnormality, suggesting that a chromosomal disruption or deletion within this region alters expression of genes crucial for normal development of craniofacial and genital structures. Closer analysis of these regions in other individuals with CHARGE association may reveal specific genes important in the pathogenesis of CHARGE, and contribute to our understanding of the relative involvement of neural crest and other cell types in craniofacial development.

\section{ACKNOWLEDGMENTS}

The authors thank the patient and his family for their support and assistance with this study. We also thank Y. Anzai for helpful discussions. DMM is a fellow in the Medical Genetics Residency program.

\section{REFERENCES}

Blake KD, Davenport SL, Hall BD, Hefner MA, Pagon RA, Williams MS, Lin AE, Graham JM, Jr. 1998. CHARGE association: an update and review for the primary pediatrician. Clin Pediatr 37:159-173.

Blake KD, Ratcliffe JM, Wyse RK. 1989. CHARGE association in two monozygous triplets. Int J Cardiol 25:339-341.

Clementi M, Tenconi R, Turolla L, Silvan C, Bortotto L, Artifoni L. 1991. Apparent CHARGE association and chromosome anomaly: chance or contiguous gene syndrome. Am J Med Genet 41:246-250.

Davenport SL, Hefner MA, Mitchell JA. 1986. The spectrum of clinical features in CHARGE syndrome. Clin Genet 29:298-310.

Devriendt K, Swillen A, Fryns JP. 1998. Deletion in chromosome region $22 q 11$ in a child with CHARGE association. Clin Genet 53:408-410.

Hall B, Blumberg B. 1988. Familial CHARGE association: affected halfsiblings through a normal mother. Am J Hum Genet 43:A54.
Hall BD. 1979. Choanal atresia and associated multiple anomalies. J Pediatr 95:395-398.

Hurst JA, Meinecke P, Baraitser M. 1991. Balanced t(6;8)(6p8p;6q8q) and the CHARGE association. J Med Genet 28:54-55.

Ignatius J, Knuutila S, Scherer S, Trask B, Kere J. 1996. Split hand/split foot malformation, deafness, and mental retardation with a complex cytogenetic rearrangement involving 7q21.3. J Med Genet 33:507-510.

Kumar A, Becker L, Depinet T, Haren J, Kurtz C, Robin N, Cassidy S, Wolff D, Schwartz S. 1998. Molecular characterization and delineation of subtle deletions in de novo "balanced" chromosomal rearrangements. Hum Genet 103:173-178.

Lopez-Rangel E, Hrynchak M, Friedman J. 1993. Cardio-facio-cutaneous (CFC) syndrome in a child carrying an inherited inversion of chromosome 7. Am J Med Genet 47:326-329.

Metlay LA, Smythe PS, Miller ME, 1987. Familial CHARGE syndrome: clinical report with autopsy findings. Am J Med Genet 26:577-581.

Mitchell JA, Giangiacomo J, Hefner MA, Thelin JW, Pickens JM. 1985. Dominant CHARGE association. Ophthalmic Paediatr Genet 6:271276.

North KN, Wu BL, Cao BN, Whiteman DA, Korf BR. 1995. CHARGE association in a child with de novo inverted duplication (14) $(\mathrm{q} 22 \rightarrow \mathrm{q} 24.3)$. Am J Med Genet 57:610-614.

Oley CA, Baraitser M, Grant DB. 1988. A reappraisal of the CHARGE association. J Med Genet 25:147-156.

Pagon RA, Graham JM Jr., Zonana J, Yong SL. 1981. Coloboma, congenital heart disease, and choanal atresia with multiple anomalies: CHARGE association. J Pediatr 99:223-227.

Tajara E, Varella-Garcia M, Gusson A. 1989. Interstitial long-arm deletion of chromosome 7 and ectrodactyly. Am J Med Genet 32:192-194.

Tellier AL, Cormier-Daire V, Abadie V, Amiel J, Sigaudy S, Bonnet D, de Lonlay-Debeney P, Morrisseau-Durand MP, Hubert P, Michel JL, Jan D, Dollfus H, Baumann C, Labrune P, Lacombe D, Philip N, LeMerrer M, Briard ML, Munnich A, Lyonnet S. 1998. CHARGE syndrome: report of 47 cases and review. Am J Med Genet 76:402-409.

Tellier AL, Lyonnet S, Cormier-Daire V, de Lonlay P, Abadie V, Baumann C, Bonneau D, Labrune P, Lacombe D, Le Merrer M, Nivelon A, Philip N, Briard ML, Munnich A. 1996. Increased paternal age in CHARGE association. Clin Genet 50:548-550. 